\title{
Supporting Expensive Physical Models With Geometric Moment Invariants to Accelerate Sensitivity Analysis for Shape Optimisation
}

Shahroz Khan

Panagiotis Kaklis

Department of Naval Architecture, Ocean and Marine Engineering, University of Strathclyde Glasgow, UK
Andrea Serani

CNR-INM, National Research Council -Institute of Marine Engineering, Rome, Italy 


\section{Motivation}

\section{Simulation-Driven Optimization (SDO)}

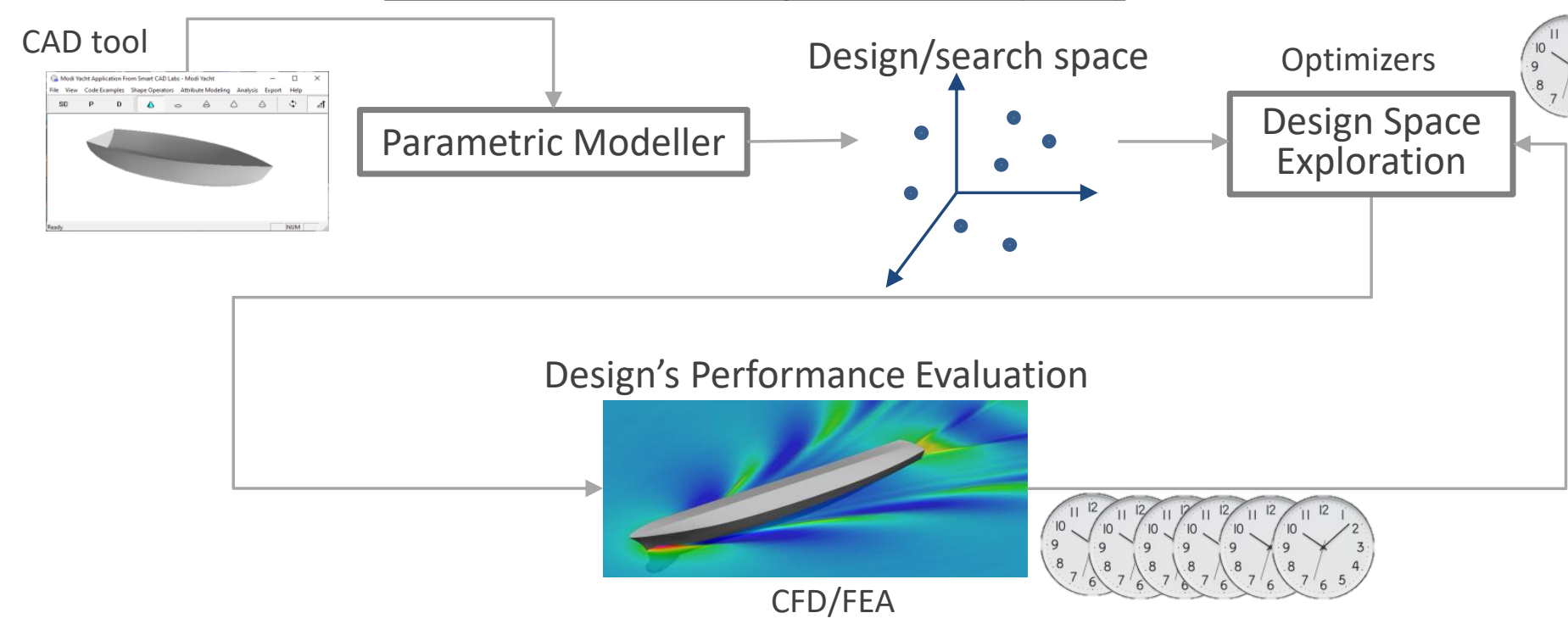

High computational cost

Rises exponentially with the dimension of the Design space 


\section{Existing Approaches}

\section{Design Space Dimensionality Reduction}

- Unsupervised - PCA, Auto-encoders Latent GEOMETRIC features

- Supervised - Sensitivity Analysis

Parameters with high variability impact on performance.

\section{Surrogate Modelling}

- Supervised - Deep/Machine Learning (PINN, NN, CNN, GAN)

Bypass the design's evaluation with CFD/FEA.
[D’Agostino et al., 2020]

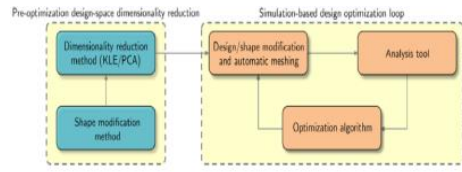

Principal Component Analysis (PCA)

[Bhatnagar et al., 2019]

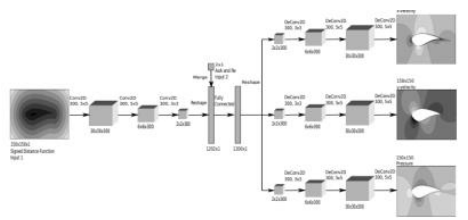

Convolutional Neural Network (CNN)
[Umetani, 2017]

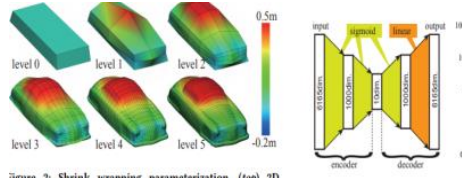

Autoencoders

[Wu et al., 2016]

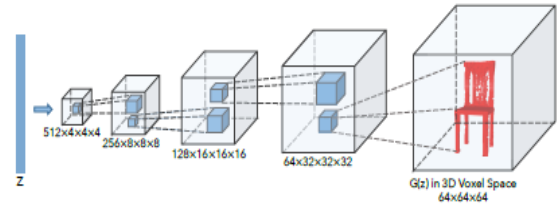

Generative Adversarial Network (GAN)

[Loua et al., 2020]

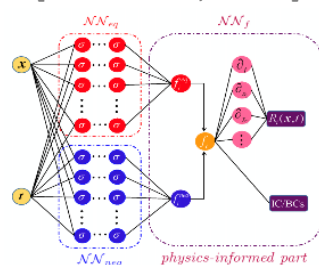

Physics Informed Neural Network (PINN) 


\section{Drawbacks}

Supervised Techniques

- Design-Space Dimensionality Reduction - sensitivity analysis

- Surrogate Modelling

Need big datasets for reliable training (High-dimensional problems)

- High fidelity simulation: single run is expensive

computational complexity still exists 


\section{Objective}

- Support physics with computationally less expensive property?

$$
\text { quantity } \approx \text { Physics and computationally less expensive }
$$

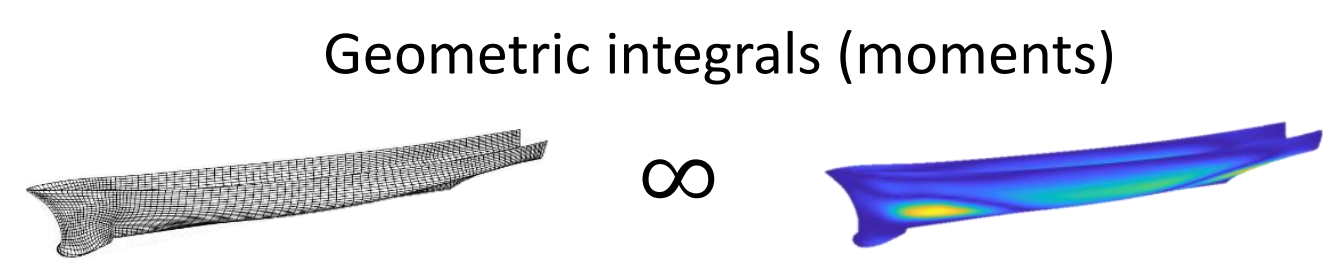

- A preliminary decision on sensitivity of parameters with geometrical properties? 


\section{Applications of Geometric Integrals (moments)}

\section{Computer-Aided Design and Computer Vision:}

- Object Recognition [Atrevi et al., 2017]

- $\quad$ Shape Retrieval [Luciano \& Hamza, 2019]

- Rigid Body Transformation [Bronstein \& Bronstein, 2018]

Geometric foundation for many physical analyses:

- Structural analysis [Kim et al., 2007]

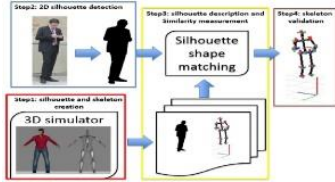

[Atrevi et al., 2017]

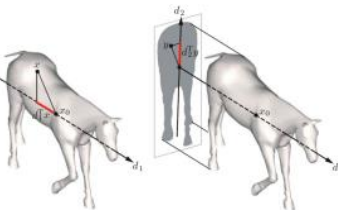

[Bronstein \& Bronstein, 2018]

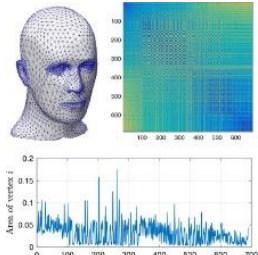

[Luciano \& Hamza, 2019]
- Meshless physical analysis [Taber et al., 2018]

- Governing equations of motion [Newman, 2008]

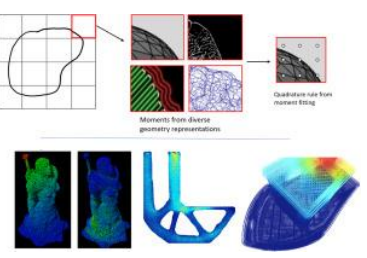

[Taber et al., 2018]

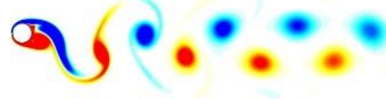

[Jin et al., 2019]
- Fluid simulations [Jin et al., 2019]

- Hydrodynamic and Hydrostatic stability [Biran \& Pulido, 2013] 


\section{Methodology - Geometric Integrals}

Geometric moments of a shape

1. are intrinsic properties of its underlying geometry

2. provide a medium for interoperability between geometry and physics.

$(l+m+n) t h-$ order moment (Riemann integrals):

$$
\begin{gathered}
M_{l m n}(\mathcal{G})=\iiint x^{l} y^{m} z^{n} \rho(x, y, z) d x d y d z \\
\rho(x, y, z)= \begin{cases}1 & \text { if } x, y, z \in \mathcal{G} \\
0 & \text { otherwise }\end{cases}
\end{gathered}
$$

Geometric domain: $\mathcal{G}$

Zeroth order moment: $M_{000}=$ volume of $\mathcal{G}$ 


\section{Methodology - Geometric Integrals}

$1^{\text {st }}$ order

$2^{\text {nd }}$ order

$\cdots$ $(l+m+n)$ th order

Moments are variant to transformation (Translation, Scaling, Rotation,)

$(l+m+n)-t h$ order central moment:

$$
M I^{l m n}(\mathcal{G})=\iiint\left(x-x_{c}\right)^{l}\left(y-y_{c}\right)^{m}\left(z-z_{c}\right)^{n} d x d y d z \quad \text { (Invariant to translation) }
$$

where $x_{c}, y_{c}$ and $z_{c}$ are the centroidal coordinates of $\mathcal{G}$

$\left(M I^{l m n}(\mathcal{G})\right.$ can be also regularised in order to become invariant to uniform scaling)

$$
\mathcal{M} \mathcal{J}^{s}=\left[\mathbf{M I}^{2}, \mathbf{M I}^{3}, \ldots, \mathbf{M I}^{\mathrm{s}}\right]
$$

shape signature vector containing all component of moments up to $s^{\text {th }}$ order

MI $^{\mathbf{s}}$ contain all moments $M I^{l m n}(\mathcal{G})$ such that $l+m+n=s$ 


\section{Methodology - Sensitive Parameters}

\section{Sensitive/Significant Parameters}

- Global Variance-Based Sensitivity Analysis (Sobol's Method)

- Sensitivity indices of each parameter w.r.t. Qol (Geometric Moments)

- Sensitive parameters: sensitivity indices $\geq$ threshold $(\varphi)$

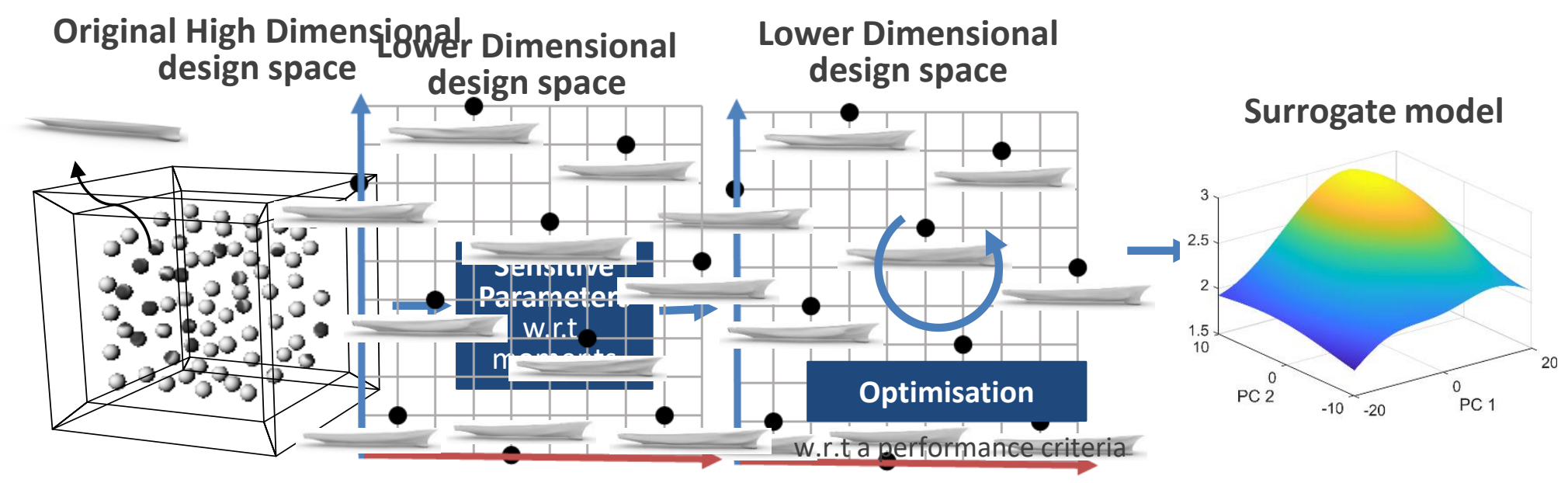




\section{Test Case}

DTMB 5415 Naval Ship Model

- Parametric modeler of GMF type depending on 27 design parameters

- GMF (global modification function) is a grid modification approach

- Objective:

Optimised design for

\begin{tabular}{|l|l|}
\hline Quantity & Value \\
\hline Displacement & $0.549 \mathrm{~m}^{3}$ \\
\hline Length between perpendiculars & $5.720 \mathrm{~m}$ \\
\hline Beam & $0.760 \mathrm{~m}$ \\
\hline Draft & $0.248 \mathrm{~m}$ \\
\hline Longitudinal centre of gravity & $2.881 \mathrm{~m}$ \\
\hline Vertical centre of gravity & $0.056 \mathrm{~m}$ \\
\hline Water density & $998.5 \mathrm{~kg} / \mathrm{m}^{3}$ \\
\hline Kinematic viscosity & $1.09 \mathrm{E}-6 \mathrm{~m}^{2} / \mathrm{s}$ \\
\hline Gravity acceleration & $9.803 \mathrm{~m} / \mathrm{s}^{2}$ \\
\hline Froude Number & 0.250 \\
\hline
\end{tabular}

calm-water wave resistance coefficient $\left(c_{w}\right)$ 


\section{Test Case}

- 27-Dimensional original design space

- Dataset Size:

9000 uniformly distributed designs - sampled with Monte Carlo method 


\section{Results - Parametric Sensitivity}

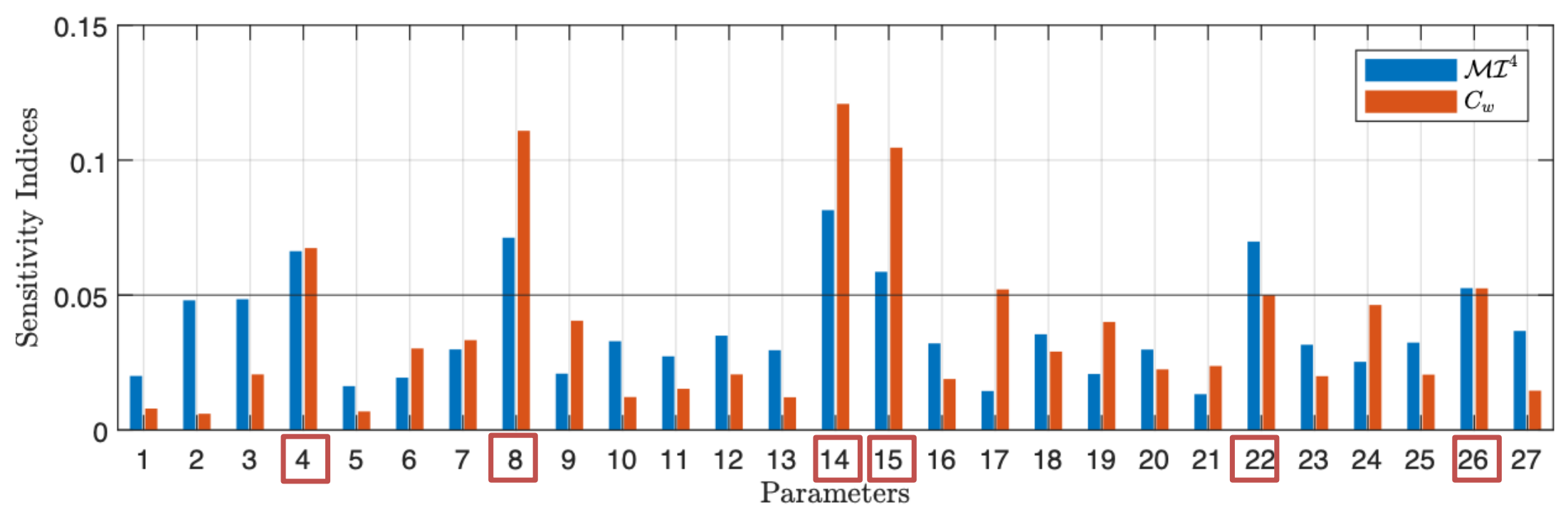

All parameters sensitive w.r.t. $4^{\text {th }}$ order moments $\left(\mathcal{M} \mathcal{J}^{4}\right)$ are also sensitive w.r.t. $c_{w}$

6 - lower dimensional design space with $\mathcal{M} \mathcal{J J}^{4}$

7 - lower dimensional design space with $c_{w}$ 


\section{Results - Optimisation}

\section{Design(\{pace \#1}

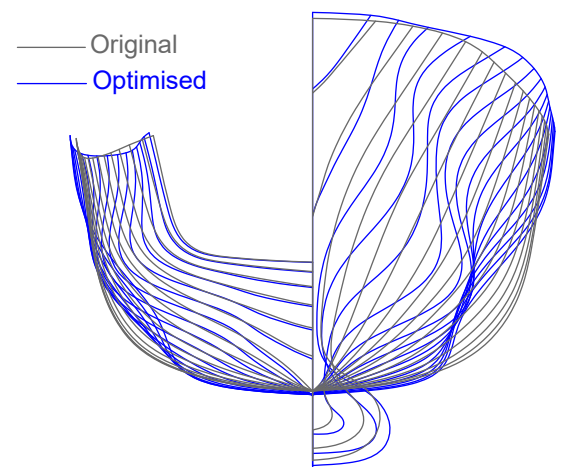

(a)

Original

Optimised (b)

Design Space \#2

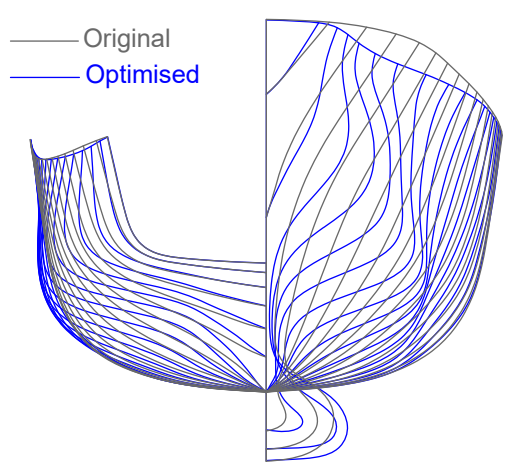

(c)

(c) with

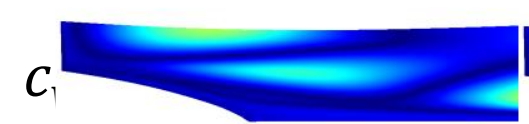

(d)

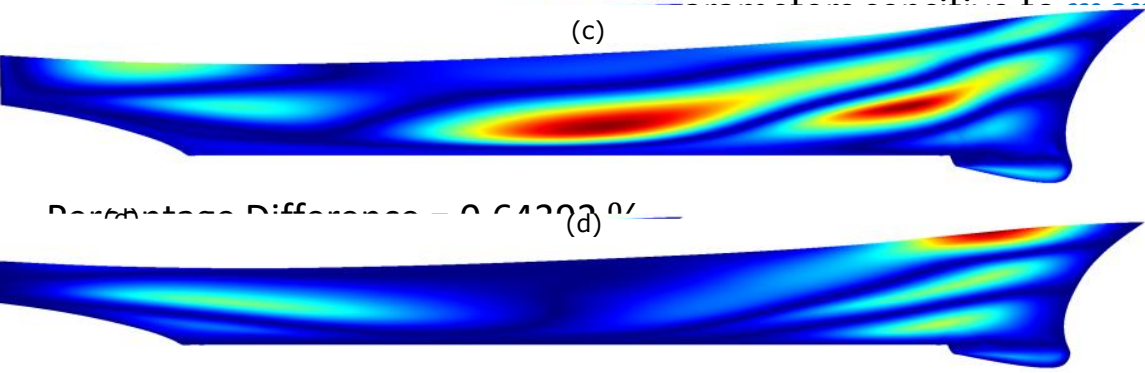

Computational cost of performing sensitivity analysis

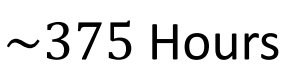

$\sim 9.5$ Hours 


\section{Conclusions \& Future Work}

Conclusion:

Moments can be used to replace/complement design's physics for parametric sensitivity analysis.

\section{Future Work:}

- New test cases with different ships, parametric modelers and solvers

- Test the influence of specific $\left(M I^{k 00}(\mathcal{G})\right)$ or higher-order $(>4)$ moments

- Investigate theoretically the correlation between moments and the physical model adopted by our solvers

- Integrate moments in surrogate modelling, especially during Physics-Informed learning. 


\section{Questions?}

\section{Funding}

University of Strathclyde

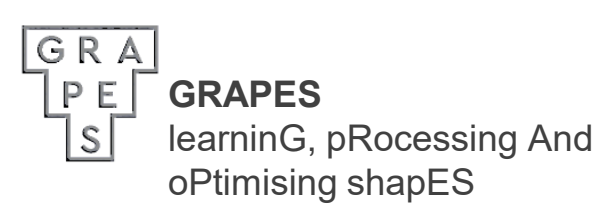

European Union's Horizon 2020 research and innovation

programme under the Marie Skłodowska-Curie grant.

\section{CNR-IN M}

US Office of Naval Research through NICOP grant N62909-18-1-2033. 